\title{
Anomalous vibrational effects in non-magnetic and magnetic Heusler alloys
}

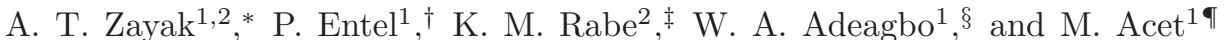 \\ ${ }^{1}$ Institute of Physics, University of Duisburg-Essen, Duisburg Campus, 47048 Germany and \\ ${ }^{2}$ Department of Physics and Astronomy, Rutgers University, Piscataway, NJ 08854-8019, U.S.A.
}

(Dated: November 7, 2018)

\begin{abstract}
First-principles calculations are used in order to investigate phonon anomalies in non-magnetic and magnetic Heusler alloys. Phonon dispersions for several systems in their cubic L $2_{1}$ structure were obtained along the [110] direction. We consider compounds which exhibit phonon instabilities and compare them with their stable counterparts. The analysis of the electronic structure allows us to identify the characteristic features leading to structural instabilities. The phonon dispersions of the unstable compounds show that, while the acoustic modes tend to soften, the optical modes disperse in a way which is significantly different from that of the stable structures. The optical modes that appear to disperse at anomalously low frequencies are Raman active, which is considered an indication of a stronger polarizability of the unstable systems. We show that phonon instability of the $\mathrm{TA}_{2}$ mode in Heusler alloys is driven by interaction(repulsion) with the low energy optical vibrations. The optical modes show their unusual behavior due to covalent interactions which are additional bonding features incommensurate with the dominating metallicity in Heusler compounds.
\end{abstract}

\section{INTRODUCTION}

In this paper, we present a detailed discussion of the electronic, magnetic and vibrational properties of Heusler compounds. This subject is of particular interest in relation to the magnetic shape-memory (MSM) effect recently discovered in these materials [1]. The Heusler compounds are known since 1903. However, only in the last decade have promising applications been presented for those systems which undergo a structural phase transformation and a magnetic phase transition near room temperature. The origin of the structural instability is related to the physics of martensitic transformations, of which the driving forces are not completely understood on a microscopic level 2]. Therefore, a detailed knowledge of the difference between structurally stable and unstable Heusler compounds on a microscopic scale would lead to a significant contribution to the theory of martensitic transformations in general and would be helpful when discussing further technical applications of MSM alloys.

With respect to technological applications, Heusler alloys are important for two reasons. Those which undergo a martensitic transformation can be used to develop mechanical devices based on the specific elastic properties of the martensitic structure, whereas the ferromagnetically ordered martensites can be used for MSM technology [4]. The MSM physics is based on the fact that the ferromagnetic martensitic structure can be deformed by applying an external magnetic field, since in MSM systems, martensitic domains are at the same time magnetic domains. It takes less energy to redistribute the

\footnotetext{
*Electronic address: zayak@physics.rutgers.edu

${ }^{\dagger}$ Electronic address: entel@thp.uni-duisburg.de

${ }^{\ddagger}$ Electronic address: rabe@physics.rutgers.edu

$\S$ Electronic address: adeagbo@thp.uni-duisburg.de

IElectronic address: macet@agfarle.uni-duisburg.de
}

(a)

(b)

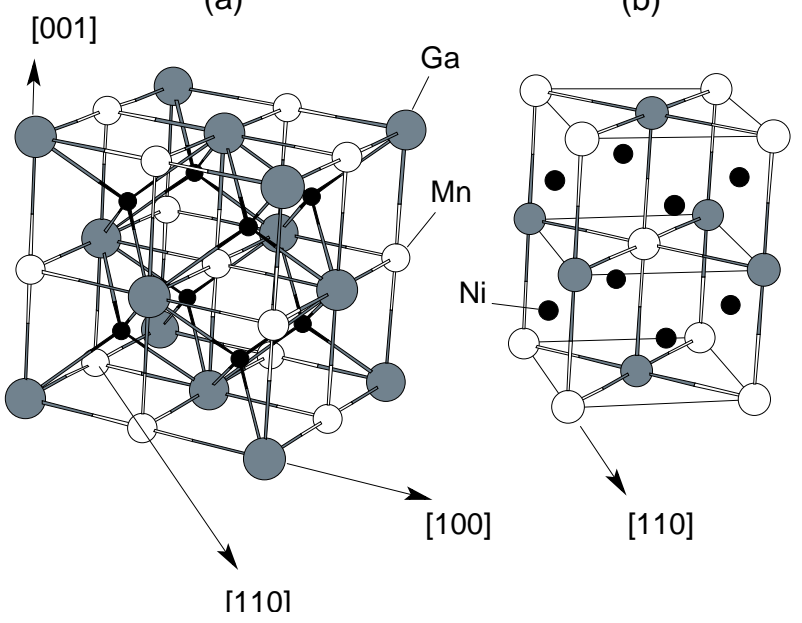

FIG. 1: (a) The L2 1 Heusler structure with unit cell of space group $\mathrm{Fm} \overline{3} \mathrm{~m}\left(\mathrm{O}_{5}^{h}\right)$ of $\mathrm{Ni}_{2} \mathrm{MnGa}$ [3] and (b) conventional tetragonal cell used in the electronic structure calculations. All compounds considered in this work have the same structure and formula unit $\mathrm{X}_{2} \mathrm{YZ}$.

martensitic domains in case of strong uniaxial magnetic anisotropy compared to the energy required to rotate the local axis of magnetization. Corresponding domains that have a favorable orientation of magnetization grow at the expense of domains for which the magnetization directions are not parallel to the external magnetic field. This has been experimentally observed in $\mathrm{Ni}_{2} \mathrm{MnGa}$ [3], which today serves as a reference system for all investigations related to MSM technology [5]. It has been shown that in moderate magnetic fields of the order of $1 \mathrm{~T}$, the structural deformations in $\mathrm{Ni}_{2} \mathrm{MnGa}$ can reach $10 \%$ [ 6 ]. This is the key feature of MSM technology that allows the design of new kinds of micro-mechanical sensors and actuators. Therefore, a complete understanding of the interplay of martensitic micro-mechanics and magnetism 
is of prime technological interest.

In this paper, we present results of investigations of the structural stability and electronic properties of the $\mathrm{L} 2{ }_{1}$ ordered non-magnetic (NM), ferromagnetic (FM) and ferri-magnetic (FerriM) Heusler compounds (the L2 1 structure is shown in Fig. 11). From this comparative study, we expect to obtain those critical parameters which distinguish unstable and stable Heusler compounds. The present analysis is a continuation of previous investigations of structural instabilities in such compounds, whereby the instability has been related to Fermi surface nesting in FM compounds [7, 8, 9]. In addition to the nesting picture, we present in this paper further unusual features of Heusler alloys which are related to characteristics of phonon dispersions and atomic displacements, electron and phonon densities of states, and the coexistence of the metallic and covalent bondings.

\section{HEUSLER ALLOYS SELECTED FOR THIS STUDY}

A set of eight Heusler alloys have been selected for this investigation. They are connected by a close relationship to $\mathrm{Ni}_{2} \mathrm{MnGa}$ which is a reference system for all studies related to the MSM properties and Heusler alloys in general, exhibiting most of the properties of interest. Moreover, we are quite familiar with this system from the previous theoretical investigations [7, 10, 11]. At compositions close to stoichiometric, this compound has the cubic L2 1 structure (see Fig. 1) which is stable at high temperature. Under cooling this crystal undergoes a series of magnetic and structural phase transitions [12, 13]. Of central interest is the soft phonon mode shown by the $\mathrm{L} 2{ }_{1}$ structure of $\mathrm{Ni}_{2} \mathrm{MnGa}$ at low temperature [14]. The character of this soft mode is believed to be related to the modulated structures $3 \mathrm{M}, 10 \mathrm{M}$ and $14 \mathrm{M}$, coupling to a uniform tetragonal distortion and the specific nesting topology of the Fermi surface of $\mathrm{Ni}_{2} \mathrm{MnGa}$ 7, 8, 10]. Similar properties have been found in other Heusler compounds [15]. By making controlled changes to the chemical composition of $\mathrm{Ni}_{2} \mathrm{MnGa}$ we plan to explore chemical trends in the electronic structure and structural energetics.

It can be helpful to characterize different Heusler compounds by their average concentration of valence electrons, which is defined as an electron-to-atom ratio [13, 16, 17]. The parameter $e / a$ is a measure of the electronic filling and allows in a rough way to distinguish in the phase diagram of the ternary alloys the range of stability for the martensitically unstable alloys. In case of $\mathrm{Ni}_{2} \mathrm{MnGa}$, $\mathrm{Ni}$ contributes ten, Mn seven, and $\mathrm{Ga}$ three valence electrons yielding $e / a=7.5$. We consider those compounds which have the same number of valence electrons as $\mathrm{Ni}_{2} \mathrm{MnGa}$ and those whose $e / a$ are slightly different. Thus, by substituting one atom of $\mathrm{Ga}$ in $\mathrm{Ni}_{2} \mathrm{MnGa}$ by atoms of $\mathrm{Al}$ or In, we obtain compounds $\mathrm{Ni}_{2} \mathrm{MnAl}$ and $\mathrm{Ni}_{2} \mathrm{MnIn}$ with $e / a=7.5$. Then, we add one valence electron in the system by substituting Ge to the place of $\mathrm{Ga}(\mathrm{Al}, \mathrm{In})$, and obtain compound $\mathrm{Ni}_{2} \mathrm{MnGe}$ with $e / a=7.75$. In $\mathrm{Ni}_{2} \mathrm{MnGa}$ and $\mathrm{Ni}_{2} \mathrm{MnGe}$, we substitute $\mathrm{Ni}$ by $\mathrm{Co}$ in order to consider compounds $\mathrm{Co}_{2} \mathrm{MnGa}(e / a=7.00)$ and $\mathrm{Co}_{2} \operatorname{MnGe}(e / a=7.25) . \mathrm{A}$ crossover from the Ni-based to the Co-based compounds is especially important due to their very different properties. The Co-based compounds are known as half-metals [18, 19]. Eventually, we obtain more or less systematic sampling of different compounds which, from preliminary analysis, are known to be ferromagnetic. In order to discuss the role of magnetic order 8] along with ferromagnets we consider one ferri-magnetic and one nonmagnetic systems, $\mathrm{Fe}_{2} \mathrm{MnGa}$ and $\mathrm{Ni}_{2} \mathrm{TiGa}$, respectively.

\section{METHOD}

\section{A. First principles calculations}

The Vienna Ab-initio Simulation Package (VASP) [20, 21] was used to perform the first-principles calculations. The projector-augmented wave formalism (PAW) implemented in this package [21, 22] leads to very accurate results comparable to other all-electron methods. The electronic exchange and correlation are treated within density functional theory by using the generalized gradient approximation [23]. The expansion of the electronic wave-functions in plane waves was done using the "High Precision" option in VASP, which corresponds to the kinetic energy cutoff as high as $337.3 \mathrm{eV}$ or more, depending on the system. For the total-energy calculations in the conventional tetragonal unit cell shown in Fig 1(b), we used a high-density mesh of $12 \times 12 \times 10$ special $k$ points for Brillouin-zone integrations. For the larger supercell used in the phonon calculations, described below, we used a $10 \times 2 \times 8$ mesh.

\section{B. Phonon calculations}

Phonon dispersions along [110] were calculated from first principles for each system in the L $2{ }_{1}$ structure at the theoretical lattice constant given in Table We used the direct force constant method [24, 25]. The supercell was a $1 \times 5 \times 1$ periodic supercell based on the conventional tetragonal cell with lattice parameters $a_{t}=b_{t}=a / \sqrt{2}$, $c_{t}=c$, shown in Fig 1 b). This is an orthorhombic supercell with the long axis along [110], containing ten consecutive (110) atomic planes along the [110] direction. We considered in turn each Cartesian displacement of each crystallographically independent atom by $0.03 \AA$ and computed the induced forces acting on all other atoms within the supercell using the Hellmann-Feynman theorem. From these results, we constructed the force constant matrix within the harmonic approximation. The phonon frequencies and corresponding eigenvectors were obtained by diagonalizing the corresponding dynamical 
matrix. The $1 \times 5 \times 1$ supercell geometry allows the direct calculation for five q-vectors along the [110] direction. These five vectors satisfy equation

$$
\exp \left(2 \pi \imath \mathbf{q}_{\mathbf{L}} \cdot \mathbf{L}\right)=1,
$$

where $\mathbf{L}$ denotes indices of the lattice constants in the supercell (in our case from 0 to 4 , giving points $\zeta=$ $0.0,0.25,0.5,0.75,1.0$, for the normalized wave vector $[\zeta, \zeta, 0]$, which spans our Brillouin zone from its center to the boundary). The interpolation of the dispersion curves is performed by setting the force constants with range beyond five atomic planes (half the length of our supercell) to zero; in the present case the decay of force constants with distance is found to be quite rapid and thus this is an excellent approximation.

\section{RESULTS}

\section{A. L2 1 structure and magnetic ordering}

For the stoichiometric Heusler compounds ( $\mathrm{X}_{2} \mathrm{YZ}$ ) considered in this work, the calculated lattice constants, magnetic moments per formula unit and the nature of magnetic ordering in the $\mathrm{L} 2{ }_{1}$ structure are given in Table [1 All atoms occupy high symmetry positions within the Fm $\overline{3} \mathrm{~m}$ (\#225) symmetry, where Wyckoff positions are 4a $(0,0,0)$ for $\mathrm{Y}$ atoms; $4 \mathrm{~b}(1 / 2,1 / 2,1 / 2)$ for $\mathrm{Z}$ atoms; $8 \mathrm{c}$ $(1 / 4,1 / 4,1 / 4)$ for $\mathrm{X}$ atoms. The computed lattice constants largely agree with the experimental values, where available, as summarized in Ref. [26, 27. The magnetic moment of $\mathrm{Fe}_{2} \mathrm{MnGa}$ is the result of local magnetic moments of $\mathrm{Mn}$ and Fe being aligned anti-ferrimagnetically. Further discussions of the magnetic properties of the Heusler alloys can be found in Ref. 28, 29.

\section{B. Calculated phonon dispersions}

The calculated phonon dispersions of the eight selected Heusler compounds in their theoretical cubic L $22_{1}$ structures are shown in Fig. 2 Some of the phonon dispersions, shown in Fig. 2 $\left(\mathrm{Ni}_{2} \mathrm{Mn}(\mathrm{Ga}, \mathrm{Al})\right)$, have already been discussed in recent publications 15, 30. Comparison of the phonon dispersions of $\mathrm{Ni}_{2} \mathrm{MnGa}$ with existing experimental data and with phonon dispersion calculations by other groups employing the linear response method show that the direct method used here yields fairly accurate results comparable to the former method [7, 31].

The [110] dispersions of the $\mathrm{L} 2{ }_{1}$ structure have one set of nondegenerate acoustic branches $\left(\mathrm{L}, \mathrm{TA}_{1}\right.$ and $\left.\mathrm{TA}_{2}\right)$ and three optical branches that can be easily recognized in Fig. 2 For the five compounds $\mathrm{Ni}_{2} \mathrm{Mn}(\mathrm{Ga}, \mathrm{Al}, \mathrm{In}, \mathrm{Ge})$ and $\mathrm{Ni}_{2} \mathrm{TiGa}$, the $\mathrm{TA}_{2}$ branch is unstable for some range of $\zeta$. In addition, in $\mathrm{Ni}_{2} \mathrm{MnGe}$ the $\mathrm{TA}_{2}$ mode has a negative slope at $\Gamma$, indicating a pure elastic instability. The instability of the $\mathrm{L} 2{ }_{1}$ structure in $\mathrm{NM} \mathrm{Ni} 2 \mathrm{TiGa}$ shows that magnetic order is not a necessary condition for the phonon softening to occur.

For some of the Heusler compounds the optical modes are split into three well separated groups which are triply degenerate at $\Gamma$, but become more mixed with increasing wave vector. In each of these three groups one finds dominating vibrations of a particular kind of atom. Mostly, this is due to the differences in the atomic masses and it can nicely be established by computing the partial phonon density of states for the [110] branch, shown for two selected compounds in Fig. 3 .

Comparison of these two plots shows that the position of the $\mathrm{Ni}$-peak in case of unstable compound $\mathrm{Ni}_{2} \mathrm{MnGa}$ is different from the position of the Co-peak in stable system $\mathrm{Co}_{2} \mathrm{MnGe}$. This was unexpected, because atoms of $\mathrm{Ni}$ and $\mathrm{Co}$, in both compounds, occupy the same sites in the structure, Wyckoff positions $8 \mathrm{c}(1 / 4,1 / 4,1 / 4)$. Their masses are close to each other. Thus, the sequence of the optical modes should be similar. However, while in case of stable compound $\mathrm{Co}_{2} \mathrm{MnGe}$ the sequence of the optical modes is normal, for the case of $\mathrm{Ni}_{2} \mathrm{MnGa}$ we observe an inversion of the optical modes.

\section{DISCUSSIONS}

\section{A. Inversion of the optical modes}

In the following we will discuss the impact of the inversion of the optical modes shown in Fig. 3 in relation to the traditionally accepted point of view of the nature of incommensurate instabilities in Heusler alloys. As a matter of fact the structural instability of the $\mathrm{L} 22_{1}$ phase is associated with Fermi surface nesting which also causes instability of the $\mathrm{TA}_{2}$ mode in some systems leading to an incommensurate product phase, which has a modulated structure. The relation between soft mode behavior and specific nesting feature of the Fermi surface has been recently emphasized and is not a debate any more [7, 8, 9].

However, a systematic review of the phonon dispersions reveals a characteristic behavior of the optical $\mathrm{T}_{2 g}$ mode at $\Gamma$ (see Fig. (4). In all Ni-based systems we find that this mode appears at much lower energies as compared to the Co-based stable compounds. The role of this anomaly might be as important as the instability of the $\mathrm{TA}_{2}$ mode, or in other words, these two features have to be considered together in order to explain the structural instabilities in Heusler alloys.

We drew attention to the fact that phonon modes of the same symmetry must repel each other. This is what must happen in case of the $\mathrm{TA}_{2}$ and the $\mathrm{T}_{2 g}$ modes. At finite wave vectors only one optical mode which has [1-10] polarization will repel with the the $\mathrm{TA}_{2}$. The main point is that there are two instabilities in the system. One of them is that the (110) planes in the Heusler structure can slide in the [1-10] direction, this is the $\mathrm{TA}_{2}$ mode. But at the same time the (111) planes of Ni slide against 
TABLE I: Computed lattice parameters, magnetic moments per unit cell and types of magnetic order for the eight selected Heusler compounds in the L2 1 structure. 'Instability' of the cubic structure means here that an unstable mode appears in the calculated phonon dispersion. The valence-electron-to-atom ratio $e / a$ for the NM compound is shown in brackets in order to avoid direct comparison with the magnetic systems in terms of this parameter.

\begin{tabular}{lccccc}
\hline \hline System & $\mathrm{a}_{\mathrm{L}_{1}}(\AA)$ & $\mu_{\text {total }}\left(\mu_{\mathrm{B}}\right)$ & L2 $2_{1}$ & Magn. order & $e / a$ \\
\hline $\mathrm{Ni}_{2} \mathrm{MnGa}$ & 5.8067 & 4.35 & unstable & $\mathrm{FM}$ & 7.50 \\
$\mathrm{Ni}_{2} \mathrm{MnAl}$ & 5.7000 & 4.20 & unstable & $\mathrm{FM}$ & 7.50 \\
$\mathrm{Ni}_{2} \mathrm{MnIn}$ & 6.0624 & 4.22 & unstable & $\mathrm{FM}$ & 7.50 \\
$\mathrm{Ni}_{2} \mathrm{MnGe}$ & 5.8039 & 4.10 & unstable & $\mathrm{FM}$ & 7.75 \\
$\mathrm{Co}_{2} \mathrm{MnGa}$ & 5.7100 & 5.09 & stable & $\mathrm{FM}$ & 7.00 \\
$\mathrm{Co}_{2} \mathrm{MnGe}$ & 5.7285 & 4.99 & stable & $\mathrm{FM}$ & 7.25 \\
$\mathrm{Ni}_{2} \mathrm{TiGa}$ & 5.8895 & 0.00 & unstable & NM & $(6.75)$ \\
$\mathrm{Fe}_{2} \mathrm{MnGa}$ & 5.6882 & 2.15 & stable & FerriM & 6.50 \\
\hline
\end{tabular}

each other also along the same [1-10] direction, and this is the $\mathrm{T}_{2 g}$ mode, shown in Fig. 团 These vibrations are destructive for each other and have to repulse. Thus, the $\mathrm{TA}_{2}$ mode is unstable because it is pushed down by the corresponding from the symmetry optical $\mathrm{T}_{2 g}$ mode of $\mathrm{Ni}$. The Fermi surface nesting determines at which wave vector the $\mathrm{TA}_{2}$ mode is most sensitive to the influence of the optical mode.

\section{B. Hybridization features}

In order to understand this observation we have to recall that Heusler alloys are very unusual metallic systems due to the presence of the so called $p$-elements $(\mathrm{Ga}, \mathrm{Al}, \mathrm{Ge}$ etc), which form partially filled bands close to $\mathrm{E}_{F}$. These bands allow the $d$ electrons of transition metals hybridize with the $p$ electrons of the $p$ element 29]. In case of $\mathrm{Ni}_{2} \mathrm{MnGa}$, the Ga atoms form energetically favorable hybrid states with Ni. These states give a peak in the spindown electronic density of states right at the Fermi level (see Fig. 5). The presence of this peak around $\mathrm{E}_{F}$ is related to the anomalous vibrational properties of the optical mode $\mathrm{T}_{2 g}$.

In contrast to this, $\mathrm{Co}_{2} \mathrm{MnGe}$ does not show a peak in the DOS close to $\mathrm{E}_{F}$. The same kind of hybridization probably occurs in this case, but the $p$ band in this case sits at lower energies so that when $d$ electrons of Co enter this band we observe what is called half-metallic behavior, i.e. there are no spin-down states at the Fermi level as can be seen in Fig. 囵

This hybridization features affect binding mechanisms in Heusler alloys to a large extent. These intermetallics and a competition of covalent interaction and magnetic ordering was already discussed by Kübler et al. in [28]. In our case, the covalent bond stems from the $4 p$ electrons of Ga which can couple to the $3 d$ electrons of $\mathrm{Ni}$. The magnetism is mainly governed by the magnetic JahnTeller effect. This effect splits the giant peak of Mn $3 d$ states, which is present in the non-magnetic DOS at the Fermi level [9]. The stability of the FM Heusler systems depends on a balance between magnetic ordering of the Mn atoms and covalent bonding of $\mathrm{Ga}$ and $\mathrm{Ni}$ atoms. However, we have shown, taking $\mathrm{Ni}_{2} \mathrm{TiGa}$ as an example, that non-magnetic Heusler compounds exhibit the same kind of martensitic instability as the FM ones and the feature in DOS is also present in this case (see Fig. [6). Therefore, the magnetic order cannot be considered to be at the origin of the structural stability. Instead, more general features of the band occupations and the hybridization have to be considered. It is important that while in metallic systems the covalent interactions are expected to be weak, the present calculations of the phonon dispersions show that some Heusler systems can exhibit strong anomalies due to the covalent bonding.

It is important that the low lying optical modes of $\mathrm{Ni}$ are the only Raman-active modes. We know from the mechanism of Raman scattering that this process must involve the polarizability of the material. This is certainly related to the covalent interaction between the Ga $4 p$ and $\mathrm{Ni} 3 d$ states. The distortion of the electron cloud will help us further to consider one more aspect of this issue because the tetrahedral environment of the atoms in the Heusler alloys leads to a natural distortion of the charge around $\mathrm{X}$ atoms (in $\mathrm{X}_{2} \mathrm{YZ} ; \mathrm{X}=\mathrm{Ni}, \mathrm{Co}, \mathrm{Fe}$ ).

Apparently, it is difficult to obtain experimental evidence of the optical modes inversion since the metallic character of our systems will wash-out any signals of the Raman activity. However, Raman-active Ni vibrations have so far been observed in an amorphous metal 33 . A Raman scattering experiment on $\mathrm{Ni}_{2} \mathrm{MnGa}$ would allow to follow up the vibrational behavior of $\mathrm{Ni}$ from the parent phase through the martensitic structure with decreasing temperature. We suggest that the anomalous sequence of the optical phonons in the structurally unstable Heusler systems is a general feature and can be applied generally to other intermetallics.

Finally, since we have involved the issue of optical 
(a) $\mathrm{Ni}_{2} \mathrm{MnGa}(\mathrm{FM})$

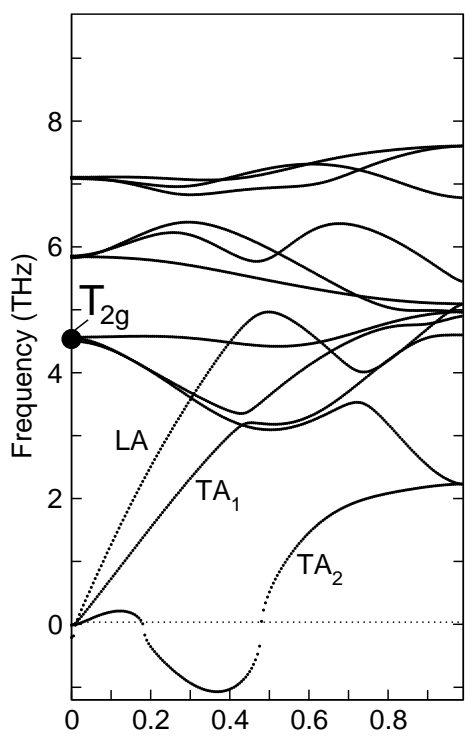

(e) $\mathrm{Co}_{2} \mathrm{MnGa}(\mathrm{FM})$

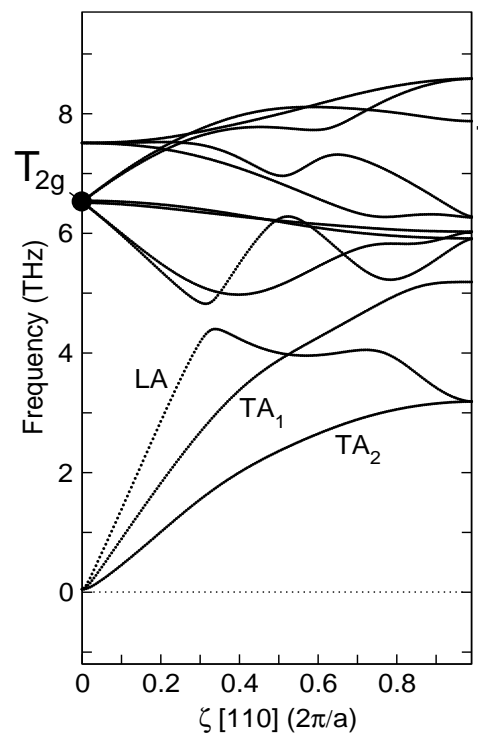

(b) $\mathrm{Ni}_{2} \mathrm{MnAl}(\mathrm{FM})$

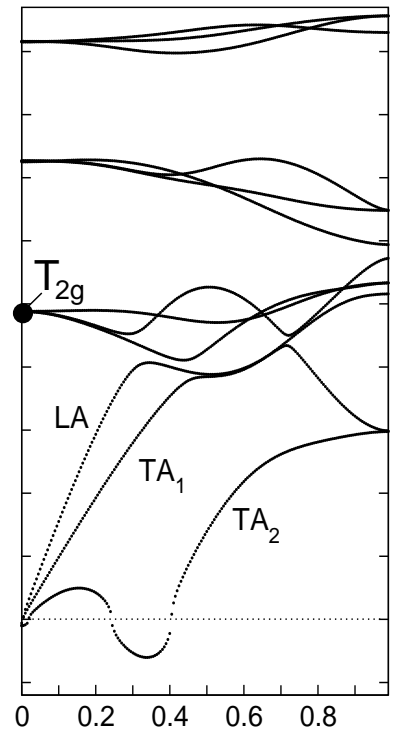

(f) $\mathrm{Co}_{2} \mathrm{MnGe}(\mathrm{FM})$

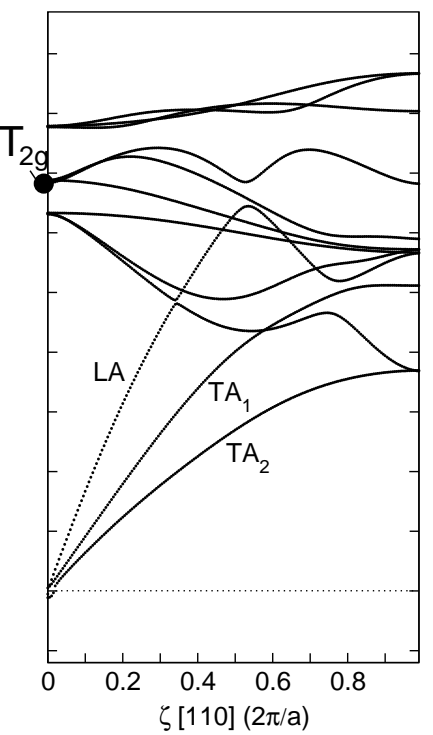

(d) $\mathrm{Ni}_{2} \mathrm{MnIn}(\mathrm{FM})$

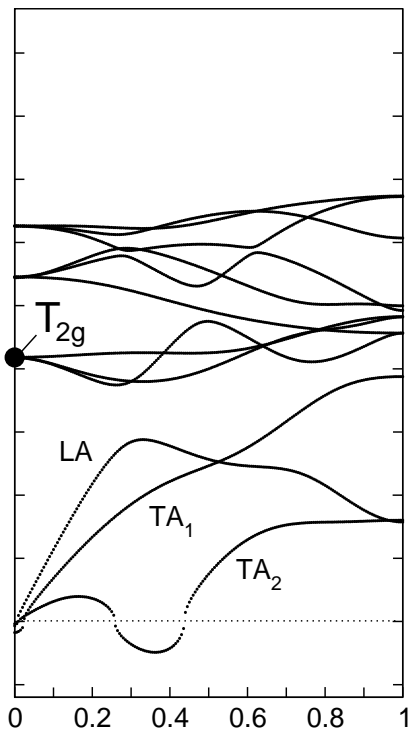

(g) $\mathrm{Ni}_{2} \mathrm{TiGa}(\mathrm{NM})$

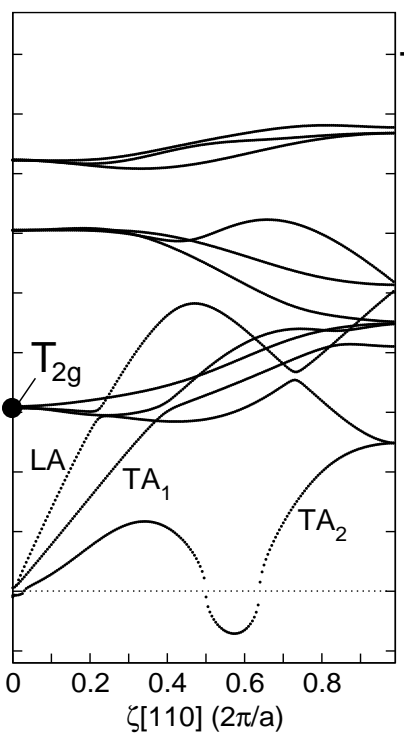

(c) $\mathrm{Ni}_{2} \mathrm{MnGe}(\mathrm{FM})$

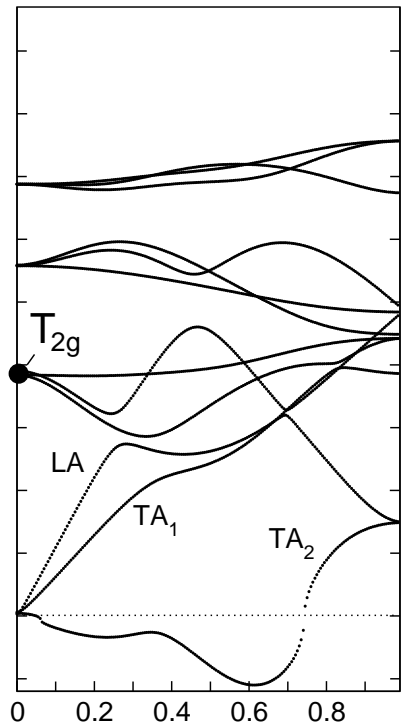

(h) $\mathrm{Fe}_{2} \mathrm{MnGa}$ (Ferri)

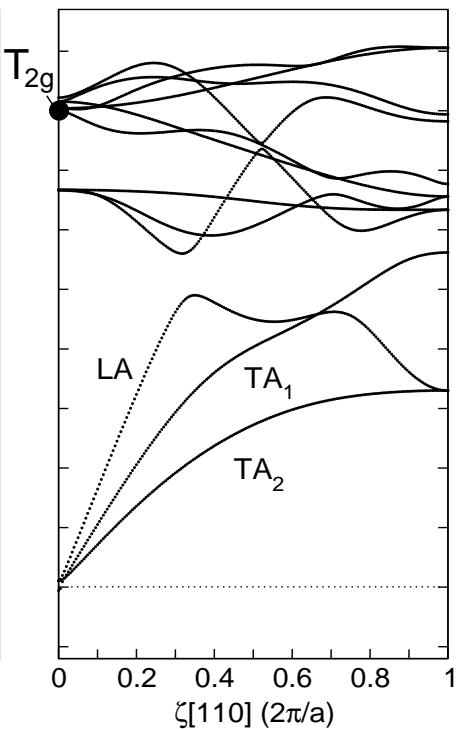

FIG. 2: Phonon dispersion curves of (a) FM Ni $\mathrm{MnGa}_{2}$, (b) FM Ni $\mathrm{FnAl}_{2}$, (c) FM Ni $\mathrm{FnGe}_{2}$, (d) $\mathrm{FM} \mathrm{Ni}_{2} \mathrm{MnIn}_{\text {(e) } \mathrm{FM} \mathrm{Co}} \mathrm{MnGa}$ (f) $\mathrm{FM} \mathrm{Co}_{2} \mathrm{MnGe}$, (g) NM Ni $2 \mathrm{TiGa}$ and (h) FerriM Fe $\mathrm{NnGa}_{2} \mathrm{Mn}$ the $\mathrm{L} 2_{1}$ structure. Here, the reduced wave vector coordinate $\zeta$ spans the $f c c$ Brillouin zone from $\Gamma$ to $X$. Imaginary frequencies of the unstable modes are shown in the real negative frequency range. The frequency of the optical modes $\mathrm{T}_{2 g}$ at $\Gamma$ is marked by a black; note it appears in lower values as compared to the stable systems.

phonons and covalent interactions in our discussions, it is worth thinking here about possible similarity with ferroelectrics, in which the structural instability can also be discussed on the basis of hybridization effects, in this case of oxygen $p$ and transition metal $3 d$ states. For the unusual behavior of phonon dispersion in ferroelectrics see, for example, 34.

\section{Trends with $\mathrm{e} / \mathrm{a}$}

The parameter $e / a$ is of rather qualitative nature and has to be discussed in relation to other details of the electronic structure discussed above. Here, we present some observations which can be useful in the development of a conceptual macroscopic view on the stability of Heusler structures.

The character of softening of the acoustic $\mathrm{TA}_{2}$ mode of 


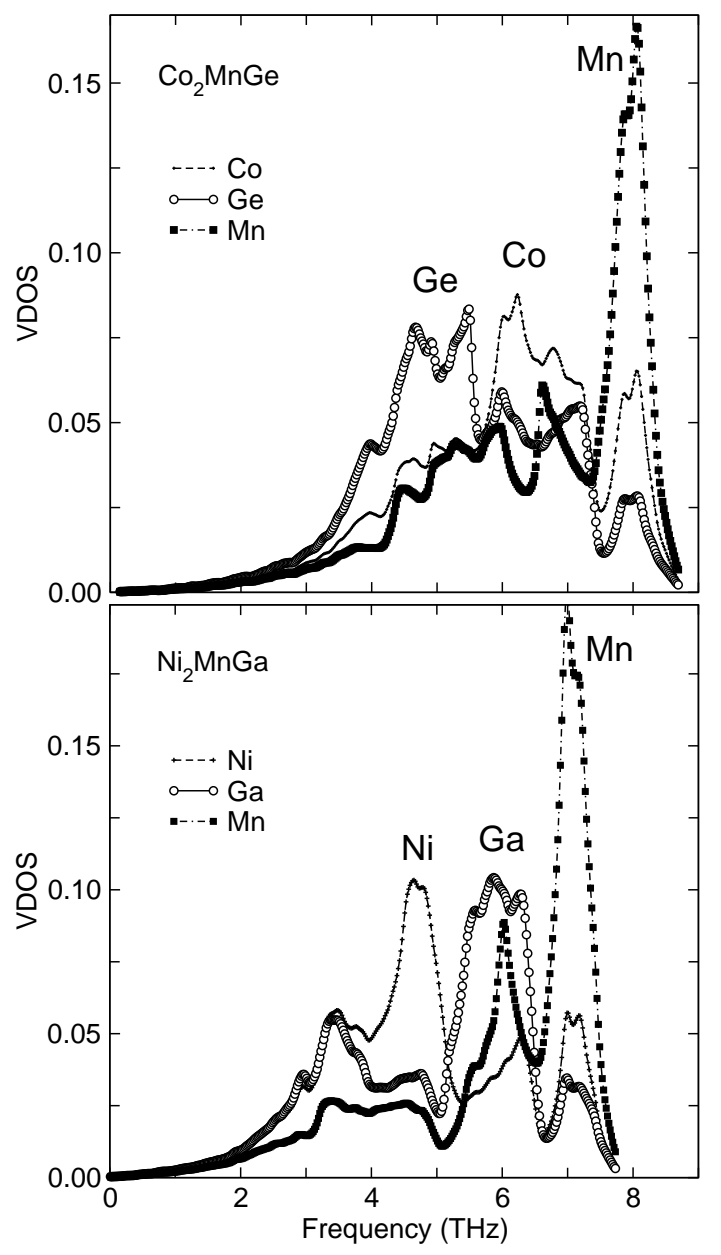

FIG. 3: Site projected vibrational density of states showing the distribution of amplitudes of the atoms over the frequency range. This information shows which atom dominates in the vibrations of a given frequency range. In particular, the anomalous behavior of optical vibrations of Ni lying at frequencies below those of the the heavier Ga atom in unstable $\mathrm{Ni}_{2} \mathrm{MnGa}$ is clearly visible (see also Fig. 2). In the case of the stable system $\mathrm{Co}_{2} \mathrm{MnGe}$, the sequence of the optical modes is regular, i.e. increasing as the mass of the atom decreases.

$\mathrm{Ni}_{2} \mathrm{MnGa}, \mathrm{Ni}_{2} \mathrm{MnAl}$ and $\mathrm{Ni}_{2} \mathrm{MnIn}$ is similar. For these compounds $e / a$ is the same, 7.5. If we compare the phonon dispersions of these systems to those having one additional valence electron, i.e. $e / a=7.75$, as in the case of $\mathrm{Ni}_{2} \mathrm{MnGe}$, we find that the onset of instability at $\zeta \approx 0.6$ is more pronounced. We do not focus here on the additional instability in the dispersion of $\mathrm{Ni}_{2} \mathrm{MnGe}$ at $\Gamma$, it has a different origin. In contrast to this unstable behavior of the Ni-based systems, the phonon dispersions of ferromagnetic Co-based stoichiometric compounds do not show any trace of a structural instability (Fig. 22). In this case, the number of valence electrons is smaller than 7.5. Thus, we see that Co- and Ni- based systems show different behavior with respect to the shear instability involving the $\mathrm{TA}_{2}$ phonon mode. Although, it is not clear

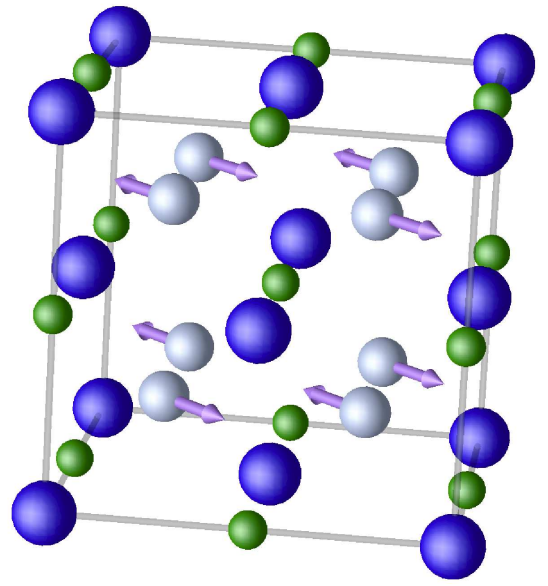

FIG. 4: (Color online) The $\Gamma$-point $\mathrm{T}_{2 g}$ optical mode which vibrates at anomalously low energy. Note that this is the only phonon mode with $\mathrm{T}_{2 g}$ symmetry in this structure and it is Raman active. Moreover, this mode is pure Ni-vibrations, of Co, i.e. the atoms sitting in the $8 \mathrm{c}$ Wyckoff positions. Other atoms are not involved in this mode. Other optical modes involve vibrations of all atoms together and have symmetry $\mathrm{T}_{1 u}$ at $\Gamma$, being IR-active.

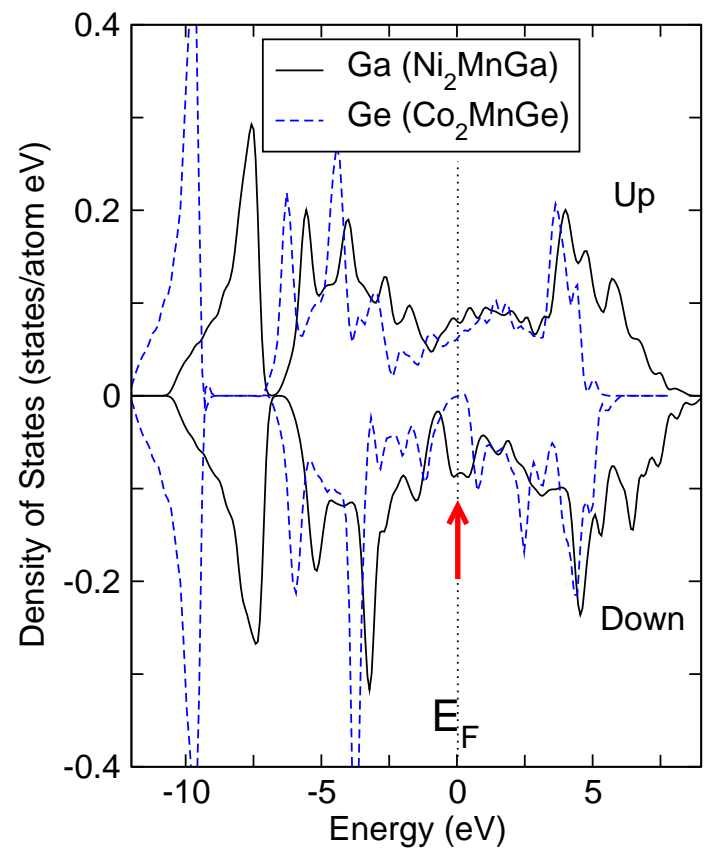

FIG. 5: (Color online) Site projected electronic density of states of $\mathrm{Ni}_{2} \mathrm{MnGa}$ and $\mathrm{Co}_{2} \mathrm{MnGe}$ in the $\mathrm{L}_{1}$ structure. There is a difference in the density of spin-down electronic states of both compounds close to the Fermi level. In $\mathrm{Ni}_{2} \mathrm{MnX}$ (X = $\mathrm{Ga}, \mathrm{Ge}, \mathrm{Al}, \mathrm{In})$ compounds, the peak in the DOS marked by the arrow arises from $p$-states of the $\mathrm{X}$ atom. The double structure of this peak is related to the hybridization splitting cause by the interaction of $4 p_{\downarrow}$ and Ni-3d $d_{\downarrow}$ states 32 . 


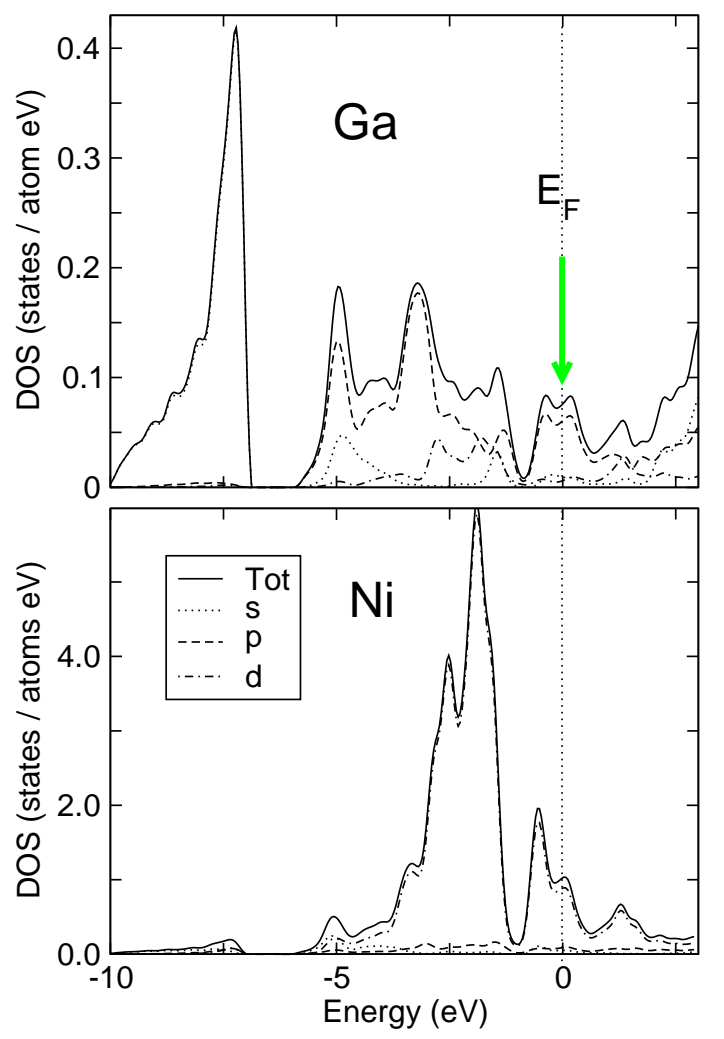

FIG. 6: (Color online) Site projected electronic density of states of $\mathrm{Ni}_{2} \mathrm{TiGa}$. The double peak structure at the Fermi level is caused by the hybridization of $\mathrm{Ga} 4 p$ and $\mathrm{Ni} 3 d$ states showing that non-magnetic Heusler systems can also exhibit the same kind of characteristic peak structure as the FM systems.

what is that key difference between $\mathrm{Co}$ and $\mathrm{Ni}$, which makes Heusler structures stable and unstable. Intrinsic properties of the $\mathrm{Ni}$ and $\mathrm{Co}$ atoms would require different kind of approach not used in this paper. It is left for future discussions in order to concentrate on other aspects of our work.

We come back to the value of $e / a$ in order to check if there is a trend which suggests a critical $e / a$ value to determine whether the $\mathrm{L} 21$ phase is stable or unstable. Maybe it is possible regardless what kind of atom (Ni of $\mathrm{Co}$ ) we have in the structure, which would be very useful for analizing alloying effects in the Heusler alloys and their stability. For this purpose we consider nearest neighbor force constants between the atoms sitting in the $8 \mathrm{c}$ Wyckoff positions i.e. Ni or Co in our case. Figure 7 presents results for only ferromagnetic systems. From this picture we can see that both for Ni- and Co- based systems the force constants follow similar trends suggesting lost of their stability at some critical values for $e / a$. Here we have two possibilities. We extrapolated lines corresponding Co- and Ni- based systems and show that thay have different behavior. Such an approach shows that replacing $\mathrm{Co}$ by $\mathrm{Ni}$ would shift the linear depen-

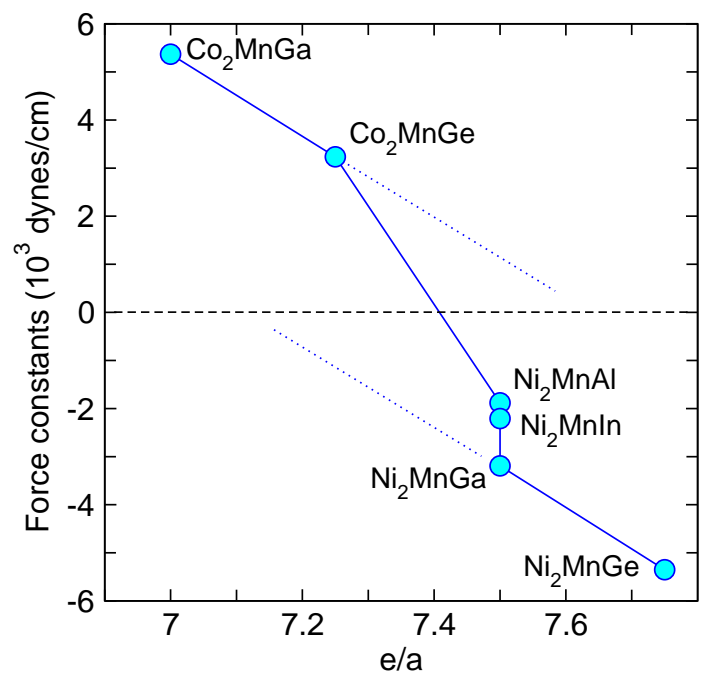

FIG. 7: (Color online) Nearest neighbor Ni-Ni and Co-Co force constants ( $\mathrm{X}$ atoms in $\mathrm{X}_{2} \mathrm{YZ}$ Heusler compounds). The force constants strongly depend on the valence electron number per atom. Possibly, there is a critical value of $e / a$ allowing the separation of structurally unstable Heusler systems with negative force constants from those of stable ones with positive force constants, but this has to be verified by additional calculations. Note that these Ni-Ni negative force constants do not lead directly to the unstable acoustic mode $\mathrm{TA}_{2}$, but it leads to the inversion of the optical modes.

dence down and other way around if we repkace Ni by Co. Contrary to that, we can assume that the kind of atom is not important, but the $e / a$ dependence of the force constants is not linear and a crossover from stable to unstable configurations occurs at around $e / a=7.4$. However, our data is not sufficient to draw any final conclusion for these two scenarios. This needs to be verified in future studies.

We would like to note that when looking at three systems, $\mathrm{Ni}_{2} \mathrm{MnAl}, \mathrm{Ni}_{2} \mathrm{MnGa}$ and $\mathrm{Ni}_{2} \mathrm{MnIn}$ with the same $e / a=7.5$ we find that the differences in their force constants are small. This shows that the size and mass of Al, Ga or In do not play much role, but the similar valence electron filling seems to be more important.

We did not include $\mathrm{Ni}_{2} \mathrm{TiGa}$ and $\mathrm{Fe}_{2} \mathrm{MnGa}$ in the Fig 7 keeping in mind that the type of magnetic order should lead to significant distinctions in how the electrons fill appropriate energy levels with possible relation to the critical ratio of $e / a$. With respect to the role of the magnetic order, it is helpful to keep in mind previous results related to the interplay of magnetic order and martensitic instability has previously been discussed for $\mathrm{Ni}_{2} \mathrm{MnGa}$ [8, 36]. It has been shown that the spin-split band structure gives rise to a prominent peak in the the wave vector dependent magnetic susceptibility close to $\mathbf{q}=\frac{2 \pi}{a}\left(\frac{1}{3}, \frac{1}{3}, 0\right)$, which corresponds to the experimentally observed wave vector for the premartensitic instability [8]. This enhanced susceptibility has its origin in 
Fermi surface nesting in the different spin channels. The fact that FM Co-based systems are stable, although tendencies of Fermi surface nesting exist, reflects an obvious situation when the characteristic topology of the Fermi surface must strongly depend on the $e / a$ ratio.

\section{Tetrahedral coordination of the covalent bonding}

In order to further elucidate the role of the covalent interaction, we have analyzed the distribution of the electronic charges in our ferromagnetic Heusler alloys. The comparison of the spin-up and spin-down densities of states has shown a clear difference in the charge distribution. The issue of the electronic hybridization becomes clear if we analyze the situation in $\mathrm{Ni}_{2} \mathrm{MnGa}$ by using a simple model. Figure 8 shows schematically the charge distribution (spin-down only) taken from ab initio calculations for $\mathrm{Ni}_{2} \mathrm{MnGa}$ in the (110) plane of the tetragonal unit cell shown in Fig. 1b. We observe an electrostaticlike repulsion between the $\mathrm{Mn}$ and $\mathrm{Ni}$ atoms. The reason for this effect is the large magnetic moments of Mn. Spindown $3 d$ electrons of Ni do not find symmetry-allowed $d$ states of Mn to hybridize with, unless they flip their spin. Instead, the $3 d$ electrons of Ni hybridize with the $4 p$ electrons of Ga. It looks like the charge of $\mathrm{Ni}$ is pushed towards the neighboring $\mathrm{Ga}$ atoms, which are tetrahedrally coordinated around it. Thus, the tetrahedral coordination which we find in the Heusler alloys is a natural basis for the $3 d$ electrons of $\mathrm{Ni}$ to hybridize with the $4 p$ electrons of Ga.

\section{CONCLUSIONS}

Our total energy density functional theory investigations of the structural instabilities in non-magnetic and magnetic Heusler compounds allow us to draw following conclusions.

We have found that in all Heusler compounds which show acoustic unstable mode $\mathrm{TA}_{2}$, the optical vibrations exhibit unusual inversion of their modes. Analysis of the force constants revealed that negative force constants are present in all unstable systems, but these force constants are not directly related to the acoustic phonon instability. Instead, the inversion of the optical modes appears to be the driving force for the acoustic anomaly. The Raman-active optical modes lower their energy due to the negative force constants, whereby the acoustic mode $\mathrm{TA}_{2}$ becomes unstable due the repulsive interaction with the anomalous optical mode. This acoustic-optical interaction might be of more general interest then just for the Heusler compounds. Its role in the martensitic transformations has to be investigated in more detail, including those in binary alloys.

A comparative analysis of different systems has shown that unstable Heusler compounds show characteristic fea-

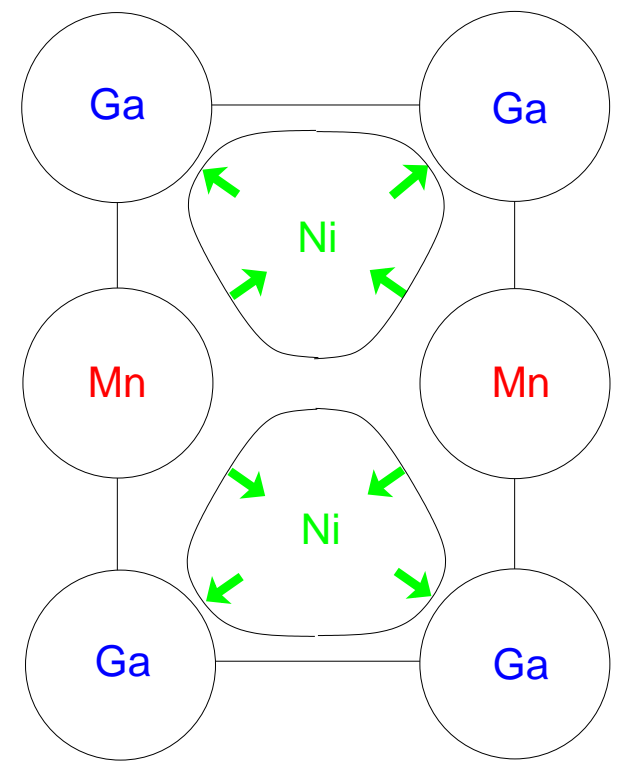

FIG. 8: (Color online) Schematic representation of the deformation of the outer $3 d$ electron cloud around the Ni atoms in the unstable Heusler alloys from present ab initio calculations. There are two effects which contribute to the deformation. One is due to covalent bonding of Ga $4 p$ and $\mathrm{Ni} 3 d$ states, the other (stronger) effect is due electrostatic repulsion of $\mathrm{Mn}$ and $\mathrm{Ni}$ atoms resulting from their nearly spherical charge distribution. The deformation of the $3 d$ orbitals of $\mathrm{Ni}$ is of tetrahedral nature and strengthens the covalent $\mathrm{Ni}-\mathrm{Ga}$ bond.

tures in the electronic DOS. The peak in the electronic states appears at the Fermi level, being responsible for the nesting topology of the Fermi surface of the unstable Heusler systems and the covalent bonding features which affect the optical vibrational modes of the Ni-based systems.

We obtained that phonon instabilities observed in the Heusler compounds can be related to the number of valence electrons in the systems. However, our consideration of only Co- and Ni-based systems does not allow us to discuss how general this observation can be. A crossover from the stable Co- to unstable Ni-based systems on the basis of the $e / a$ value can be suggested, but additional investigations are required. Microscopic intrinsic properties of $\mathrm{Co}$ and $\mathrm{Ni}$ atoms may stand for a substantial part of our observations.

Study of additional Heusler compounds (see Ref. 27) will serve to further establish physical trends to the problem of martensitic phase transitions.

\section{ACKNOWLEDGEMENTS}

This work was supported by SFB-491 (Germany) and NSF MRSEC DMR-00-80008 (U.S.A.). ATZ thanks E. Liskova, K. Parlinski, A. Postnikov and C. Fennie for 
very helpful and inspiring discussions.

[1] I. Takeuchi, O. O. Famodu, J. C. Read, M. A. Aronova, K.-S. Chang, C. Craciunescu, S. E. Lofland, M. Wuttig, F. C. Wellstood, L. Knauss, et al., Nature Mater. 2, 180 (2003).

[2] E. F. Wassermann, Privat communications (2004), Duisburg.

[3] P. J. Webster, K. R. A. Ziebeck, S. L. Town, and M. S. Peak, Phil. Mag. 49, 295 (1984).

[4] K. Ullakko, J. K. Huang, C. Kantner, R. C. O'Handley, and V. V. Kokorin, Appl. Phys. Lett. 69, 1966 (1996).

[5] S. J. Murray, M. A. Marioni, A. M. Kukla, J. Robinson, R. C. O'Handley, and S. M. Allen, J. Appl. Phys. 87, 5774 (2000).

[6] A. Sozinov, A. A. Likhachev, N. Lanska, K. Ullakko, and V. K. Lindroos, J. Phys. IV (France) 112, 955 (2003).

[7] C. Bungaro, K. M. Rabe, and A. D. Corso, Phys. Rev. B 68, 134104 (2003).

[8] Y. Lee, J. Y. Rhee, and B. N. Harmon, Phys. Rev. B 66, 054424 (2002).

[9] O. I. Velikokhatnyi and I. I. Naumov, Phys. Solid State 41, 617 (1999).

[10] A. T. Zayak, P. Entel, J. Enkovaara, A. Ayuela, and R. M. Nieminen, J. Phys.: Condens. Matter 15, 159 (2003).

[11] A. T. Zayak and P. Entel, Mater. Sci. Eng. A 378, 419 (2004).

[12] V. V. Martynov and V. V. Kokorin, J. Phys. III (France), 2, 739 (1992).

[13] L. Dai, J. Cullen, and M. Wuttig, Journal of Applied Physics 95, 6957 (2004).

[14] A. Zheludev, S. M. Shapiro, P. Wochner, A. Schwartz, M. Wall, and L. E. Tanner, Phys. Rev. B 51, 11310 (1995).

[15] T. Büsgen, J. Feydt, R. Haßdorf, S. Thienhaus, M. Moske, M. Boese, A. Zayak, and P. Entel, Phys. Rev. B 70, 014111 (2004).

[16] V. A. Chernenko, Scripta Materialia 40, 523 (1999).

[17] V. Chernenko, V. L'vov, E. Cesari, J. Pons, and
R. Portier, Materials Transactions 43, 856 (2002).

[18] W. E. Pickett and J. S. Moodera, Physics Today 54, 39 (2001)

[19] I. Galanakis, P. H. Dederichs, and N. Papanikolaou, Phys. Rev. B 66, 134428 (2002).

[20] G. Kresse and J. Furthmüller, Phys. Rev. B 54, 11169 (1996).

[21] G. Kresse and D. Joubert, Phys. Rev. B 59, 1758 (1999).

[22] P. E. Blöchl, Phys. Rev. B 50, 17953 (1994).

[23] J. P. Perdew, K. Burke, and Y. Wang, Phys. Rev. B 54, 16533 (1996).

[24] K. Parlinski, Z. Q. Li, and Y. Kawazoe, Phys. Rev. Lett. 78, 4063 (1997).

[25] K. Parlinski, PHONON, Cracow, Poland (2002).

[26] K. Ziebeck and P. Webster, in Landolt-Börnstein New Series (Springer-Verlag, 1988), vol. III/19c, p. 75.

[27] K. Ziebeck and K.-U. Neumann, in Landolt-Börnstein New Series (Springer-Verlag, 2001), vol. III/32c, p. 64.

[28] J. Kübler, A. R. Williams, and C. B. Sommers, Phys. Rev. B 28, 1745 (1983).

[29] I. Galanakis, P. Dederichs, and N. Papanikolaou, Phys. Rev. B 66, 174429 (2002).

[30] A. T. Zayak, P. Entel, J. Enkovaara, A. Ayuela, and R. M. Nieminen, Phys. Rev. B 68, 132402 (2003).

[31] A. Zheludev, S. M. Shapiro, P. Wochner, and L. E. Tanner, Phys. Rev. B 54, 15045 (1996).

[32] A. Ayuela, J. Enkovaara, and R. M. Nieminen, J. Phys.: Condens. Matter 14, 5325 (2002).

[33] N. Lustig, R. Fainchtein, and J. S. Lannin, Phys. Rev. Lett. 55, 1775 (1967).

[34] P. Ghosez, E. Cockayne, U. V. Waghmare, and K. Rabe, Phys. Rev. B 60, 836 (1999).

[35] F. R. Vukajlovic, E. L. Shirley, and R. M. Martin, Phys. Rev. B 43, 3994 (1991).

[36] A. Planes, E. Obrado, A. Gonzales-Comas, and L. Manosa, Phys. Rev. Lett. 79, 3926 (1997). 